Copyright (2015) John Benjamins Publishing Company. The publisher should be contacted for permission to re-use or reprint the material in any form. This article is published as Vallee-Tourangeau, Frederic (2014) Insight, materiality and interactivity. Pragmatics \& Cognition, 22(1), pp. 27-44 and may be found at http://dx.doi.org/10.1075/pc.22.1.02val.

\title{
Insight, interactivity and materiality
}

\author{
Frédéric Vallée-Tourangeau \\ Kingston University
}

The popular iconography of insight casts a thinker as he or she uncoils from a Rodin pose and a bulb that lights a world hitherto hidden. By and large, these features of folk mythology capture and guide how psychologists conduct research on insight: Mental processes - some of which may be unconscious - transform an inceptive abstract representation of the world until it prescribes a fruitful solution to a problem. Yet thinking and problem solving outside the laboratory involve interacting with external resources, and through this interactivity with a material world, solutions are distilled. Still, laboratory work on problem solving pays scant and largely indifferent attention to interactivity: Sometimes problems are presented as riddles or static graphical or diagrammatic images, or sometimes they are accompanied by artefacts that can be manipulated (and sometimes interactivity is possible for some problems but not others within a set of problems over which performance is indiscriminately amalgamated). The research methodology - and indifference to the central role of interactivity in thinkingfollows from a deep-seated commitment to mentalism and methodological individualism. However, a thinker is an embodied creature embedded in a physical world: The materiality of external resources and artefacts through which problems manifest themselves inevitably determines a set of action affordances. From a systemic perspective, thinking is traceable along a contingent spatio-temporal itinerary wrought by interactivity and evidenced by changes in the world.

Keywords: Insight, interactivity, materiality, affordance

\section{Insight, interactivity and materiality}

Insight holds special fascination among folk psychologists and the scientific cognoscenti alike. The impasse head scratch, the light bulb flash and the phenomenological lightness that accompanies a breakthrough triangulate a popular conception that has also informed psychological research. For the past 100 years, psychologists have sought to capture, describe and explain insight. While some of those original efforts were informed by fieldwork (albeit with chimpanzees [Köhler 1925]), by and large psychologists have sought to investigate insight under laboratory conditions. Clearly, this creates a number of challenges in mapping what goes on inside the lab to the purported phenomena of interest outside, but this is no less true of the biologists or the chemists plying their science in the lab.

I open the review of problem-solving research with the contrast often made between so-called insight and analytic problems and briefly describe the types of processes that have been conjectured to play a central role in the transformation of an initial representation of the problem into one from which a solution can be derived. Researchers agree that, on the whole, solving insight problems involves restructuring: a re-interpretation of the problem that casts 
its elements and their relations in a light that identifies new paths to solution. This proposal is supported and guided by a number of interrelated theoretical and ontological assumptions and concomitant methodological prescriptions. That is, restructuring is a mental phenomenon driven by mental processes decoupled from the world. Since this is a mentalist story, "representational change processes do not correspond to any particular overt behaviors" (Ohlsson 2011: 113). As a consequence, problem-solving psychologists often conduct experiments in a manner that pays scant and inconsistent attention to the physical presentation of the problem and the participants' opportunity to manipulate the physical elements of the problem. Fleck and Weisberg (2013; Weisberg 2015) recently published a theoretical framework to account for the range of mental processes that may be implicated in problem solving. They based their framework on verbal protocol data. Rather tellingly, the two protocols prominently showcased in the 2013 paper involved a participant manipulating the physical presentation of the problem. The authors made inferences about the nature and timing of the restructuring of the participant's mental representation of the problem, based on the participant's actions that modified the physical features of the problem. The role of interactivity was completely ignored by Fleck and Weisberg. I show that in fact, in their data, restructuring was much more likely when participants could physically modify a problem's physical presentation, and I question the possibility and ultimate usefulness of identifying separate types of restructuring processes, namely, conceptually driven and data driven.

Ignoring a problem's physical presentation and the potential for interactivity betray a deep misconception of thinking outside the psychologist's laboratory. There, thinking is the product of a fluid and dynamic interaction with external resources that produces a shifting configuration of physical features and action affordances. I next review recent work that illustrates the importance of designing experimental procedures that foster interactivity and demonstrate how problem solving is substantially transformed as a result. Interactivity weaves a contingent spatio-temporal itinerary that is shaped, guided and constrained by the material employed in the physical presentation of the problem. I close the paper with some observations on the usefulness of the distinction between analytic and insight problem types and how restructuring should be documented in the laboratory.

\section{Restructuring and insight problems}

Problem-solving researchers have examined how people work on two broad classes of problems: insight and analytic problems. While hard-and-fast membership criteria remain elusive, it is generally the case that, for analytic problems, the goal state is clearly understood and reconcilable with the initial problem presentation. In addition, the operators that can transform proto-solutions into the goal state are readily identifiable and easily accessible. For example, multi-digit number multiplications like 746 times 859 can be coolly completed with pencil and paper by employing simple operators. When tallying up the intermediate calculations to 640,814, jubilation rarely ensues. The Tower of Hanoi and river-crossing puzzles are analytic problems commonly employed in problem-solving research (e.g., Simon 1975; Knowles and Delaney 2004). The goal state is clearly defined, the mapping of the initial problem information to the goal state is largely unproblematic (e.g., move all the disks from the left-most peg onto the right-most peg), the problem space of transitional states is fully specifiable and moves in that space are actionable with simple operators. What interests psychologists with analytic problems is the nature of the move-selection strategy and the efficiency with which participants traverse the problem space (sometimes as a function of 
learning - repeated experience - and transfer to superficially different but structurally isomorphic problems).

In contrast, insight problems have goal states that initially appear irreconcilable with the given information. In addition, the operators needed to transform progressive approximations of the goal state are unknown. Thus, solving insight problems does not reflect a "gradual accretion of learning" (Hebb 1949: 160). In the laboratory, insight problems often take the form of riddles. For example, Luo and Niki (2003) presented participants with the statement, "The thing that can move heavy logs, but cannot move a small nail" (Luo and Niki 2003: 317). Participants must shift their interpretation of the problem in a manner that helps them focus on the density of the objects named rather than their weight, in the hope of deriving 'river' as the answer. Thus, insight problems are designed to create an impasse, and a range of dependent variables - verbal protocols, latency to solution, moves to completion, neuroimaging - offer a window onto the mental processes that evince a change to, or a restructuring of, the interpretation of the problem into a new one that clearly identifies a solution. Theorists from different perspectives (e.g., Ohlsson 1984; Weisberg 1995; Fleck and Weisberg 2004; Ohlsson 2011) share a common goal: to identify and measure the nature of these mental processes. Some conjecture that these involve largely unconscious recoding and reinterpretation of the problem elements or the relaxation of assumptions that constrain the range of possible solutions to the problem (e.g., Ohlsson, 1984, 1992, and Ohlsson's [2011] more recent redistribution theory). The restructured problem representation coalesces into consciousness, unbidden, corresponding to the breezy suddenness of insight sometimes reported. Others bet on conscious analytic examination of the problem features. The initial stages of this analysis may yield a solution without participants having to restructure their interpretation of the problem. However, a persistent impasse state will necessitate some form of restructuring, although again this may be gradually developed on the basis of conscious analysis (Fleck and Weisberg 2013; Weisberg 2015). Whether the processes are unconscious or not, and whether insight is experienced all at once or builds up more gradually, theorists focus their efforts on "the thinker's representation of the problem" (Weisberg 1995: 168) and the mental processes that transform this representation.

\section{Theoretical assumptions and methodological prescriptions}

In this section, I outline two influential empirical contributions to the psychology of insight problem solving originating from research teams whose efforts share fundamental ontological assumptions and common methodological prescriptions, despite differences in their respective theoretical agendas. First, Knoblich, Ohlsson, Haider and Rhenius (1999) designed a series of matchstick arithmetic problems using Roman numerals with which to evaluate the role of constraint relaxation in restructuring a problem representation. Participants were shown a false expression like 'IV = III + III' made up of 'matchsticks' and asked to movenot remove - one matchstick in order to turn the expression into a true one. Since numerals are generally the first thing that participants think of modifying with such problems, decomposing them leads to a swift solution. Other problems are much harder to solve; for example, the expression 'III = III + III' requires relaxing the conversational constraint of avoiding tautologies - the solution is ' $\mathrm{III}=\mathrm{III}=\mathrm{III}$ ' - as well as the constraint on decomposing operators. Indeed, Knoblich et al. observed much lower solution rates for these problems. The relative success rate was interpreted in terms of constraint relaxation that drove the restructuring of the mental representation of the problem. Why speak of mental representations if participants can simply 'play' with these models? Couldn't the world be 
restructured without the guidance of a mental representation that was restructured first? Well, one of the reasons why this possibility was not entertained by the authors was the fact that these matchstick problems involved no actual matchsticks and thus no interaction with a physical presentation of the problem. The arithmetic expressions were presented on a computer screen, and participants looked at them until they announced their answers to an experimenter. In a subsequent eye-tracking experiment using the same problems (Knobich et al. 2001), immobility was additionally ensured by putting a bite bar in participants' mouths: Problem solving happens inside the head, so better keep it still. As I show later, interactivity matters a great deal in solving matchstick algebra problems.

In a recent paper, Fleck and Weisberg (2013) used verbal protocol data to identify the nature of the processes implicated in problem solving. Participants were trained to narrate their thinking as they worked on a problem. The coding of these verbal protocols provided data used to identify and classify different analytic strategies and search heuristics, and to determine whether participants' mental representation of the problem was restructured. The theoretical framework that emerged from these data specified a range of problem-solving methods, from a direct transfer of a solution, through an analysis of the problem features. Different heuristics, such as means-end analysis, could guide the process, which may then have yielded a restructuring of the problem representation. In this instance, the restructuring reflected the participant's mental efforts and was termed 'conceptually driven'. If the participant was unable to change his or her representation of the problem, restructuring may have been brought about by chancing on a change in the problem presentation; the restructuring was then termed 'data-driven'.
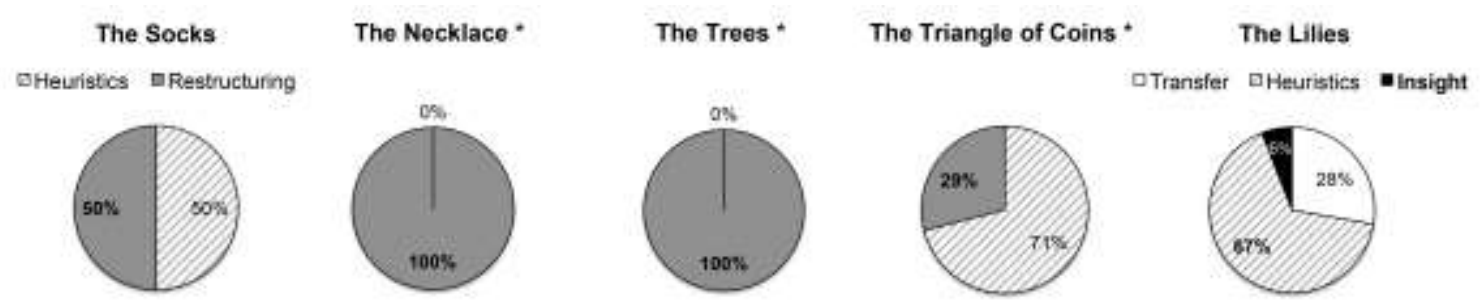

Figure 1. Problem-solving methods for successful problem solvers for each of the five problems used in Fleck and Weisberg (2013). Note that problems annotated with asterisks (namely, The Necklace, The Trees and The Triangle of Coins) were presented with artefacts. Adapted from Table 3 in Fleck and Weisberg (2013: 451).

The verbal protocols were obtained as participants tackled a series of five problems. ${ }^{1}$ Two of the problems were presented without artefacts, the others as written descriptions supplemented with artefacts that configured the problem. Participants were free to manipulate the artefacts and reconfigure the problem as they saw fit as they worked on a solution. The prevalence of the different methods employed to reach a solution for all five problems is illustrated in Figure 1. Restructuring was apparent in most problems, but particularly so when artefacts were provided. This was noted in the description of the data by the authors (Fleck and Weisberg 2013: 450) but attracted no additional commentary.

Fleck and Weisberg (2013) defined data-driven restructuring as "instances when the individual changed his or her representation of the problem in response to something he or she saw from the physical configuration of the problem" (452). Later the authors noted that data-driven restructuring was "stimulated by a configuration of the problem materials produced unintentionally during exploration, rather than from directed thinking or reflection 
on the problem by the problem solver" (Fleck and Weisberg 2013: 453). The 'unintentional' qualifier is interesting: action without thinking - chancing upon contingent problem configurations-likely plays an important role in 'real-world' problem solving. In turn, conceptually driven restructuring "[comes] about as a result of change in the participant's thinking about the problem goal or constraints, in response to information arising from unsuccessful work on the problem, without impasse" (Fleck and Weisberg 2013: 453-454). The distinction suggests that these different sources of restructuring can be isolated in the dynamic interplay between thought, action and perception fostered in a context where participants can reconfigure the physical presentation of the problem. As I argue later, what is restructured, and what should be the starting point of any analysis of successful and unsuccessful problem solving, is the world. A science of problem solving should be focused on how the world is restructured during problem solving, and an initial-first orderdescription of the actions that fashion this restructuring process need not be anticipated by a mental presentation of the problem. In addition, the fluid transaction between thought, action and the world makes it not only difficult to isolate different sources of restructuring but calls into question the ontological plausibility of this independent segmentation.

It is interesting to note that the two protocols cited at length by Fleck and Weisberg (2013) — in fact, the only protocols provided in the paper-were recorded as the participant worked on the solution of a problem that was also presented with manipulable artefacts: Interactivity was the substrate from which the narrative emerged. The first protocol was offered as an illustration that problem solving can proceed without restructuring (the participant was working on The Triangle of Coins problem); what did change, as illustrated in the protocol, was the physical presentation of the problem. The authors in fact illustrated through a six-panel figure (Fleck and Weisberg 2013: 453-454) how the physical presentation was modified through interactivity:

I'm just moving them [the coins] around, trying to see how it's gonna work. [Figure 4B: Moves the bottom point coin to side of the 3-coin row making four; then moves this coin to the top in the centre.] There's not as many on the bottom as on the top. 4, $3,2,1 ; 3,2,1 ; 2,1 ; 1$. Yeah, I can't just move them from the bottom; I have to move them from the top too. [Figure 4C: Moves top left corner coin down, next to the 3coin row to make 4 . Then sets the coins in the beginning problem configuration; Figure 4D.] So move this one up [Figure 4E: moves bottom coin to the top centre]. Trying to one, two, three [Figure 4F: moves top right and left corner coins down to bottom 2-coin row to make 4]. OK? (Fleck and Weisberg 2013: 452)

On the basis of this verbal protocol and in the absence of a more detailed qualitative analysis of the problem-solving trajectory, it is very difficult to isolate the mental planning from the acting. In addition, to speak of the absence of restructuring in light of the dramatic changes to the physical appearance of the problem seems to ignore a fundamental feature of problem solving: Insight is wrought by interactivity. To the extent that this is a representative protocol, the rate of restructuring might be substantially underestimated in these data.

The other protocol cited at length by Fleck and Weisberg (2013) is one that illustrates what the authors term conceptually driven restructuring (the participant is working on The Trees): 
So, 10 trees in 5 straight lines. That doesn't sound possible. [Participant arranges the trees in 2 straight lines with 5 trees in each.] It doesn't seem like you could do it in straight vertical and horizontal lines so I guess I have to put in on a diagonal. [Participant arranges the trees in 2 diagonal lines that intersect to form an X.] ... You're gonna use 5 straight lines, but you need to use like each tree in more than one. So that would be 2 lines.... Make a triangle pattern. Would that work? [Arranges the trees like a triangle with 3 lines of 4 trees in each.] That would be 3 lines. We still need more. Need 2 more lines. So, wait, wait, wait. You start with a point and have a ray. Checkerboard? Diagonals? I have a feeling the diagonals are part of this. You kind of need to have as many lines as possible while still like reusing the same, the same, um, points.... You could make a star shape. [Participant draws a star on the paper and then places the trees on it.] (Fleck and Weisberg 2013: 454).

Fleck and Weisberg (2013) argue that the restructuring documented here is conceptually driven because the physical reconfiguration appears to follow an observation-a case of planning and then acting (O'Hara and Payne 1998). Fleck and Weisberg proceed on the basis of an implicit linear decompositionality premise (Baber et al. 2014), that planning and acting in the dynamics of problem-solving activity can be segmented orthogonally. Furthermore, Fleck and Weisberg's argument suggests that conceptually driven restructuring is a process of imposing form on matter - a hylomorphic model of creativity (Ingold 2009) rather than one where actions on and with the material world weave new forms that may not have been anticipated before the actions are initiated, what Ingold (2009) refers to as the 'textility of making'. In the participant's narrative, the perceptual feedback and the very act of playing around with the artefacts may seed the next observation and shape the next inference, the exact timing and dynamic interweaving of which is not reflected in the verbal protocol data (as presented in the paper). I would argue that without a more detailed analysis of the timing and interweaving of the verbal narrative with the participants' movements, it is difficult to draw with any precision a distinction between conceptually driven and data-driven restructuring.

The importance of interactivity in driving restructuring is not a focus of Fleck and Weisberg's (2013) work; it gets no mention and is not the subject of any analysis. However, there is evidence in their paper that restructuring is much more likely in high-interactivity contexts. For example, from the information in their Table 2 (Fleck and Weisberg 2013, 450), across successful and unsuccessful problem solvers, 67\% of the participants experienced restructuring when working on the problems with artefacts, but only $18 \%$ did so for the problems without the artefacts. If one only looks at the successful participants, the presence of artefacts leads to a greater number of solutions through restructuring, with data-driven restructuring — according to the authors' definition—being more frequent (see Figure 2). 

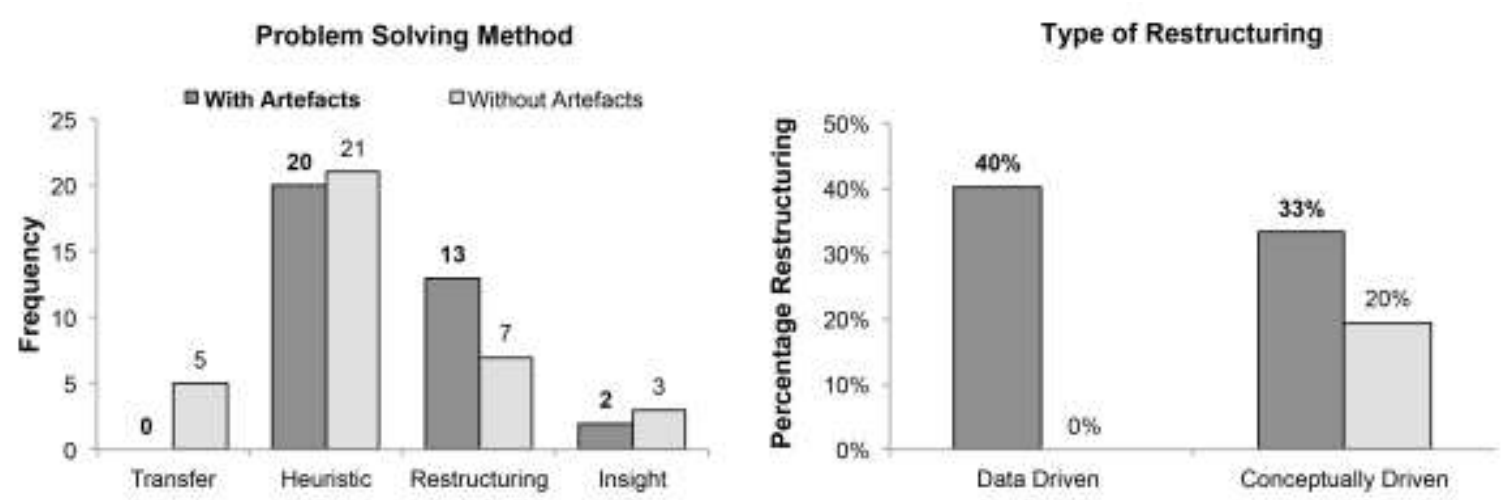

Figure 2. Frequency of solution methods for the three insight problems with artefacts and the two insight problems without artefacts (left panel); mean percentage of data-driven and conceptually driven restructuring solutions for the insight problems (with and without artefacts, right panel). Adapted from Table 3 in Fleck and Weisberg (2013: 451).

It is thus striking to note how the analyses reported in Knoblich et al. (1999) and Fleck and Weisberg (2013) ignore interactivity and its central role in thinking. The methodology employed - and in the case of Fleck and Weisberg's research, the data analyses themselves - reflect a strong commitment to a set of classical cognitivist assumptions, the core of which casts thinking as an internal computational process. The decoupling of the thinker from his or her world is a form of methodological sequestering (Vallée-Tourangeau and Vallée-Tourangeau 2014), itself predicated on methodological individualism (Malafouris 2013). Those classical assumptions not only imply that thinking can be understood by eliminating interactivity and corralling the mind, but they also foster a science focussed on the agent, rather than on the system configured by the agent embedded in a material and malleable world.

Thus, ignoring interactivity is understandable from a classical cognitivist perspective, since problem solving proceeds from a mental representation of the world. Once the world is represented internally, its role becomes peripheral and serves, at best, as a memory aid to internal processes. But the world is not simply a source of information, nor is a thinker a passive - or sometimes harnessed!-information processor. The methodological exigencies that eliminate interactivity in order to provide a more transparent window onto mental processes are misguided, unnecessary and reflect a profound misconception of problem solving.

\section{Interactivity and materiality}

When I got to our office ... I quickly cleared away the papers from the desk top so that I would have a large, flat surface on which to form pairs of bases... Though I initially went back to my like-like prejudices, I saw all too well that they led nowhere... [I] began shifting the bases in and out of various other pairing possibilities. Suddenly I became aware that a $[\mathrm{A}-\mathrm{T}]$ pair held together by two hydrogen bonds was identical in shape to a $[\mathrm{G}-\mathrm{C}]$ pair held together by at least two hydrogen bonds. All the hydrogen bonds seemed to form naturally; no fudging was required to make the two types of base pairs identical in shape. [...] Upon his arrival 
Francis did not get more than halfway through the door before I let loose that the answer to everything was in our hands (Watson 1968: 152-154; emphasis added).

This is a remarkable and telling passage in many respects. It describes the moment of discovery of how the base pairs hold the double helix. By his own admission, James Watson did not think himself a great chemist (he once quipped that he would "never be the brightest in the room" [Watson 2005]). At one point in the race to discover the nature and structure of DNA, he cut "accurate representations of the bases out of stiff cardboard" (Watson 1968: 152) because metal models took too long to manufacture, and the following day, he spent time moving cardboard shapes of the bases to form different pairs. ${ }^{2}$ Were all Watson's moves premeditated? Were some movements very swift, a reflection of action-perception loops, some mulled over longer? Were some arrangements produced because he was simply 'playing' with the shapes? Did a fortuitous arrangements cue a more insightful one? It is impossible to formulate answers for these questions from the published accounts of that day in 1953. Moving the cardboard shapes reflected the qualities and possibilities of the thinkerartefact system physically configured over Watson's desk at the Cavendish Laboratory, and interactivity was the "ontological substrate" (Steffensen 2013: 196) from which the discovery took shape. It is plausible to conjecture that Watson's appreciation of what he was seeing was prompted by his movements, and that the movements were in part guided by his interpretation of the emerging configurations. It makes little sense to segment this loop and decouple the agent form the world.

Interactivity in the psychologist's laboratory. It is important to engineer problemsolving environments in the laboratory that permits interaction with a physical presentation of a problem. We revisited the matchstick arithmetic problems developed by Knoblich and colleagues (Weller et al. 2011). This time, however, we used short magnetized sticks $(0.5 \mathrm{~cm}$ $\times 2.5 \mathrm{~cm}$ ) with which participants constructed each false expression on a small magnetic board $(21 \mathrm{~cm} \times 27 \mathrm{~cm})$. Compared to a control group that inspected a static image depicting the false Roman numeral arithmetic expression and announced their answer to the experimenter, participants in the high-interactivity condition were more likely to discover the solution for these problems, including the difficult tautological ones. Interactivity mattered in this task because moving one stick - whether that move was motivated by a plan or notchanged the visual feedback that cued a different set of moves. In other words, interactivity spawned a shifting set of action affordances. For example, given the expression 'I = II + II', picking up the vertical stick that makes the plus operator created a new visual configuration ('I = II - II; I') that cued a new set of possible actions. In turn, the unchanging visual feedback from a static display forced reasoners to simulate possible movements in their heads; the resulting mental projections were continuously vexed by conflicting visual feedback. Interactivity thus enabled the implementation of what Kirsh (2009) referred to as the project-create-project cycle.

Interactivity distills and weaves cognitive products along a contingent spatio-temporal path. To understand the genesis of insight, it becomes important to record and analyse this path. In the Systemic Cognition Laboratory at Kingston, we engineer interactive problemsolving tasks, and then film and code the participants' actions and the dynamic shifts in the problem presentation. Quantitative and qualitative analyses are performed to document the itinerary to insight. The artefacts employed in the problem presentation inevitably shape the interactivity afforded by the cognitive ecosystem configured by the reasoning agent and the physical space in which reasoning is enacted. In a recent experiment, we used the 17 animals problem (adapted from Metcalfe and Wiebe 1987), which asks participants to figure out how 
to place 17 animals in four enclosures in such a manner that there is an odd number of animals in each enclosure (Vallée-Tourangeau, Steffensen, Vallée-Tourangeau and Makri, in press). This problem masquerades as an arithmetic puzzle, but the solution involves the design of enclosures to create overlapping sets wherein animals can be placed in more than one set. All participants in the experiment labored on an arithmetic solution in an initial three-minute pencil-and-paper solution phase. Thus, all our participants experienced an impasse, and we were poised to capture the development of the overlapping-set solution, to capture the genesis of insight.

Following the initial three-minute phase, some of the participants were assigned to a 3D model-building condition, provided with pipe cleaner pieces of various lengths, animal figurines (zebra shaped paper clips) and given 10 minutes to build a model of the solution on a table surface $(118 \mathrm{~cm} \times 74 \mathrm{~cm})$. These participants were not given pencil or paper, and the problem solving could only be enacted through the model-building activity.

On the basis of a detailed coding of the video segments-some lasting only milliseconds - using the cognitive-event analysis developed by Steffensen (2013), we were also able to capture, for example, how the chenille bristle that wraps around the metal pipe cleaners sometimes gripped other pieces, and hence the manipulation of one pipe cleaner could unintentionally cause other pipe cleaner pieces to move. Thus, in the manufacturing of an enclosure or in the movement of an enclosure, a surreptitious overlap sometimes emerged as a result of a series of actions, creating an 'event pivot' that rerouted the subsequent solution itinerary (Steffensen, Vallée-Tourangeau and Vallée-Tourangeau, 2015). Only a detailed qualitative analysis of the videos could reveal how the material properties of the artefacts employed shaped the model-building actions that determined the participants' ability to solve the problem. Despite the voluminous brain-in-a-vat research produced by psychologists and neuroscientists working on insight, problem solving never occurs in a vacuum; it takes place in a cognitive ecosystem (Hutchins 2010), and insight is inextricably linked to the dynamic spatio-physical properties of that ecosystem.

External representations, representatives and interactivity. The artefacts constructed by Watson represented molecules, but it is plausible to argue that when he moved the molecules on his desk top, he was not manipulating representations, he was manipulating representatives of the molecules (Noë 2012). His movements thus directly shaped the arrangement of the base pairs, not representations of the base pairs. Participants in our 17 animals experiment did not interact with external representations but rather with a model of the world, not unlike how scientists treat molecular models as the representative of the 'real' thing and engage in a form of pretend play, the way children do (Toon 2011).

In an important paper, Zhang and Norman (1994) used the Tower of Hanoi to demonstrate how the features and rules of the problem could be embedded in the physical characteristics of the artefacts employed in different versions of the problem. Some of the rules were 'externally represented' in that they constrained participants' behavior without the participants having to rehearse mentally the rules of the problem. The Tower of Hanoi is an analytic problem with a clearly specifiable problem space that lends itself particularly well to the superlatively clear and formal treatment of external representations offered by Zhang and Norman. While some of these ideas pertinently inform a systemic perspective on thinking, I think it misses a central component of problem solving. At the heart of the matter is interactivity and how thinking is distilled through it. This fundamental aspect of thinking is ignored. Zhang and Norman measured the impact of external representations through latency and moves to completion, but this only offered a limited perspective on how participants solved the problems. Problem solving is instantiated in real time, through the continuous 
interplay of mind, hands and artefacts; without more information about the temporal trajectory enacted with different Tower of Hanoi isomorphs, we get only a limited understanding of how those problems are solved. In addition, by using an analytic problem with a very clear goal state and a small set of operators, participants know what the solution looks like; they're not looking for a solution but for the means to achieve it quickly with the fewest moves. With many real-world problems, a problem solution takes shape along a certain contingent trajectory wrought by interactivity with external resources.

Artefacts that constitute the physical model of the problem guide and constrain the manner with which problem-solving behaviour is enacted. This is a keystone proposition that unlocks unbounded heuristic value to help researchers engineer new experimental procedures and better identify the reason for the success or failure of participants at a given problemsolving task. Take for example the simple matchstick arithmetic problem: Matchsticks of different colours would alter the perceptual information from which different actionperception loops may weave unique solution paths. Or consider a board with random or fluctuating magnetic properties, such that some matchsticks would be easier to manipulate than others, some of the time. The physical and perceptual characteristics of the artefacts would configure extended cognitive systems (Wilson and Clark 2009) within which idiosyncratic solution trajectories may be enacted. The nature of the individual problem solver, transitional contingent results (Järvilehto 1998), and the affordances of the materiality of the task determine problem solving trajectories.

\section{A science of systemic problem solving}

Problem-solving research traditionally proceeds from a distinction between productive and reproductive thinking formulated by gestalt psychologists (e.g., Wertheimer 1959). Thus, reproductive thinking reflects the application of well-learned rules and schemas to new problems. As such, the thinking is not considered creative or novel, and solutions don't innovate; they replicate. In contrast, productive thinking represents a discontinuity, a substantial break from the past; there lies the genuinely creative form of thinking, the true insight.

The distinction between analytic and insight problems is generally aligned with these different forms of thinking, with researchers working on insight problems documenting productive rather than reproductive thinking. Analytic problems like the Tower of Hanoi are characterised in terms of a formally defined and fully specifiable problem space: Thinking is fundamentally reproductive in the repeated application of simple operators to move from the initial problem presentation to its desired goal state. Insight problems, however, involve productive thinking because their solution requires the development and application of a novel method to solve the problem, one that is not clearly specified in the initial problem presentation. A taxonomy of insight problems has been developed (Weisberg 1995) to help researchers identify 'pure' insight problems as a means to ensure that the measuring instruments capture authentically productive thinking.

Such a taxonomy is not without merit or usefulness. However, elaborating classification criteria independent of the context in which reasoning takes place is ultimately unproductive. From a systemic perspective, thinking reflects a dynamic transactional agentenvironment coupling. Interactivity is the engine that weaves the transactional fabric (Ingold 2009). By transactional, I mean that neither the agent nor the environment can be independently specified without considering the dynamic coupling: The agent is transformed 
by the environment, which in turn is shaped by the agent. The linear decompositionality assumption that informs theoretical frameworks like the one proposed by Fleck and Weisberg (2013) sheds a misleading light on the dynamics of real-time problem solving (Baber et al. 2014). Productive and reproductive thinking may well result from this interactivity at different points along the problem-solving path traced by the evolving configuration of the system as either an analytic or insight problem is solved. In characterising the agentenvironment system, continuities or discontinuities may be prompted by certain agentenvironment configurations. In the 17 animals problem, different stages or transitional results in the model building of the solution may encourage more reproductive thinking, or may steer a substantial change of course to the problem-solving itinerary. The evolution of agentenvironment configurations is inescapably contingent: A taxonomy of different types of thinking or different kinds of problem will never be as fruitful as a detailed analysis of the system.

Restructuring the world first. A systemic perspective casts a different light on efforts to determine whether insight problems can be solved with or without restructuring. The verbal protocol data from Fleck and Weisberg (2013) were interpreted as reflecting the presence or absence of restructuring, as well as distinguishing between data-driven and conceptually driven restructuring. I have argued that in the absence of a detailed analysis of the participants' actions as they unfold in time, these interpretations remain wanting. Be that as it may, Fleck and Weisberg's research focus makes sense only from an individualist, internalist perspective. In contrast, problem solving in the world proceeds on the basis of changes in the world: People and researchers alike act on the world, manipulate artefacts and models and rearrange physical features of a problem. This is problem solving. The evidence of successful problem solving can be found in changes in the world. Whether a mental representation was restructured or not is secondary to the behavioural and physical evidence: How interactivity exploits and modifies external resources should first be documented. The physical and behavioural evidence should be measured, analysed and understood. In the Fleck and Weisberg data (see Figure 2), restructuring was more frequently recorded when problems were presented with manipulable artefacts; data-driven restructuring was only observed with interactivity. I have argued that some instances of conceptually driven restructuring likely reflected changes in the world rather than the anticipation of the changes - the proffered verbal protocols are insufficient to determine the precedence of mental over physical restructuring. That is, on the basis of the verbal protocols, it is difficult to establish the timing and exact interweaving of narrative and action so as to establish whether actions were premeditated or whether they were cued by fluid changes to the physical arrangement of the features of the problem.

The methodological implications of a systemic perspective are clear. Much important research on problem solving can be conducted under laboratory conditions. Real-world cognitive ecosystems must be scaled down, to be sure, but problems must be presented to participants in such a manner as to permit the manipulation and rearrangement of the elements that define the problem. Eliminating interactivity will close the window onto natural problem solving, and the resulting science will be fundamentally misaligned with how human beings think. The material nature of the artefacts should also be carefully considered and itself be the subject of experimental manipulation, since it determines the action affordances that the physical model offers. The contingent spatio-temporal itinerary must be recorded and analysed to trace the genesis of insight.

These so-called transient extended cognitive systems (TECS; Wilson and Clark 2009) are assembled fluidly as people reason and solve problems. The meshwork of internal and external resources that configures a TECS is dissolved when a task is accomplished or 
assembled differently in the face of an impasse. Under laboratory conditions, the contingent nature of these TECS does not limit the science of problem solving to a historical description of their assembly and the problem-solving itinerary. A mix of quantitative and qualitative analyses can identify recurrent patterns of behaviour that help explain (and predict) why some participants solve a problem, and why others don't. A systemic science of problem solving is better positioned to explain insight.

\section{Notes}

* I would like to thank Gaëlle Vallée-Tourangeau for her thoughtful comments on a previous version of this manuscript.

1. Here are the problem descriptions as presented to the participants:

The Socks: If you have black socks in your drawer mixed in the ratio of 4:5, how many socks will you have to take out to be sure of having a pair of the same colour? The Necklace: A woman has four pieces of chain. Each piece is made up of three links. She wants to join the pieces in to a single closed ring of chain. To open a link costs 2 cents and to close a link costs 3 cents. She has only 15 cents. How does she do it? The Trees: How can you plant 10 trees in five rows with four trees in each row? The Triangle of Coins: The triangle point to the bottom of the page. How can you move only three coins and make the triangle point to the top of the page? The Lilies: Water lilies double in area every 24 hours. At the beginning of the summer, there is one water lily on a lake. It takes 60 days for the lake to become completely covered with water lilies. On what day is the lake half covered? (Fleck and Weisberg 2013: 446-447).

2. A reconstruction of the creation and manipulation of the cardboard shapes, acted by Watson himself, can be seen at http://www.dnalc.org/view/15492-Discovering-thedouble-helix-structure-of-DNA-James-Watson-video-with-3D-animation-andnarration.html.

\section{References}

Fleck, J.I. and Weisberg, R.W. 2004. "The use of verbal protocols as data: An analysis of insight in the candle problem". Memory \& Cognition 32(6): 990-1006.

Fleck, J.I. and Weisberg, R.W. 2013. "Insight versus analysis: Evidence for diverse methods in problem solving". Journal of Cognitive Psychology 25(4): 436-463. DOI: $10.1080 / 20445911.2013 .779248$

Hebb, D.O. 1949. Organization of Behavior. New York: John Wiley and Sons.

Hutchins, E. 2010. “Cognitive ecology”. Topics in Cognitive Science 2(4): 705-715. DOI: 10.1111/j.1756-8765.2010.01089.x

Ingold, T. 2009. "The textility of making”. Cambridge Journal of Economics 34(1): 91-102. DOI: $10.1093 / \mathrm{cje} / \mathrm{bep} 042$ 
Järvilehto, T. 1998. "The theory of the organism-environment system: I. Description of the theory". Integrative Physiological and Behavioral Science 33(4): 321-334. DOI: 10.1007/BF02688700

Kirsh, D. 2009. "Projection, problem space and anchoring". In Taatgen, N.A. and van Rijn, H. (eds), Proceedings of the 31st Annual Conference of the Cognitive Science Society. Austin, TX: Cognitive Science Society, 2310-2315.

Knoblich, G., Ohlsson, S., Haider, H. and Rhenius, D. 1999. "Constraint relaxation and chunk decomposition in insight problem solving". Journal of Experimental Psychology: Learning, Memory, and Cognition 25(6): 1534-1556. DOI: 0278$7393 / 99 / \$ 3.00$

Knoblich, G., Ohlsson, S. and Raney, G.E. 2001. "An eye movement study of insight problem solving". Memory \& Cognition 29(7): 1000-1009.

Knowles, M.E. and Delaney, P.F. 2005. "Lasting reductions in illegal moves following an increase in their cost: Evidence from river crossing problems". Journal of Experimental Psychology: Learning, Memory, and Cognition 31(4): 670-682. DOI: 10.1037/0278-7393.31.4.670

Köhler, W. 1925. The Mentality of Apes. London: Penguin Books.

Luo, J. and Niki, K. 2003. "Function of hippocampus in 'insight' of problem solving". Hippocampus 13(3): 316-323. DOI: 10.1002/hipo.10069

Malfouris, L. 2013. How Things Shape the Mind: A Theory of Material Engagement. London: The MIT Press.

Metcalfe, J. and Wiebe, D. 1987. "Intuition in insight and noninsight problem solving". Memory \& Cognition 15(3): 238-246. DOI: 10.3758/BF03197722

Noë, A. 2012. Varieties of Presence. Cambridge, MA: Harvard University Press.

O'Hara, K.P. and Payne, S.J. 1998. "The effects of operator implementation cost on planfulness of problem solving and learning". Cognitive Psychology 35(1): 34-70. DOI: $10.1006 / \operatorname{cogp} .1997 .0676$

Ohlsson, S. 1984. "Restructuring revisited II: An information processing theory of restructuring and insight". Scandinavian Journal of Psychology 25(2): 117-129. DOI: 10.1111/j.1467-9450.198.4.tb01005.x

Ohlsson, S. 1992. "Information-processing explanations of insight and related phenomena". In Keane, M.T. and Gilhooly, K.J. (eds), Advances in the Psychology of Thinking. Hemel Hempstead: Harvester Wheatsheaf, 1-44.

Ohlsson, S. 2011. Deep Learning: How the Mind Overrides Experience. New York: Cambridge University Press.

Simon, H.A. 1975. "The functional equivalence of problem solving skills". Cognitive Psychology 7(2): 268-288. DOI: 10.1016/0010-0285(75)90012-2

Steffensen, S.V. 2013. "Human interactivity: Problem-solving, solution probing and verbal patterns in the wild". In Cowley, S.J. and Vallée-Tourangeau, F. (eds), Cognition Beyond the Brain: Computation, Interactivity and Human Artifice. London: SpringerVerlag, 195-221. 
Steffensen, S.V., Vallée-Tourangeau, F. and Vallée-Tourangeau, G. 2015. "Cognitive events in a problem-solving task: Qualitative methods for investigating interactivity in the 17 animals problem". Manuscript submitted for publication.

Toon, A. 2011. "Playing with molecules". Studies in History and Philosophy of Science 42(4): 580-589. DOI: 10.1016/j.shpsa.2011.08.002

Vallée-Tourangeau, G. and Vallée-Tourangeau, F. 2014. "The spatio-temporal dynamics of systemic thinking". Cybernetics and Human Knowing 21(1-2): 113-127.

Vallée-Tourangeau, F., Steffensen, S.V., Vallée-Tourangeau, G. and Makri, A. (In press). "Insight and cognitive ecosystems". Proceedings of the 37th Annual Conference of the Cognitive Science Society. Austin, TX: Cognitive Science Society.

Watson, J.D. 1968. The Double Helix. London: Penguin.

Watson, J.D. 2005. "How we discovered DNA". TED talk. Retrieved from https://www. youtube.com/watch? $\mathrm{v}=2 \mathrm{HgL} 5 \mathrm{OFip}-0$

Weisberg, R.W. 1995. "Prolegomena to theories of insight in problem solving: A taxonomy of problems". In Sternberg, R.J. and Davidson, J.E. (eds), The Nature of Insight. Cambridge, MA: The MIT Press, 157-196.

Weisberg, R.W. 2015. "Toward an integrated theory of insight in problem solving". Thinking \& Reasoning 22(1): 5-39. DOI: 10.1080/13546783.2014.886625

Weller, A., Villejoubert, G. and Vallée-Tourangeau, F. 2011. "Interactive insight problem solving". Thinking \& Reasoning 17(4): 429-439. DOI: 10.1080/13546783.2011. 629081

Wertheimer, M. 1959. Productive Thinking. New York: Harper and Brothers.

Wilson, R.A. and Clark, A. 2009. "How to situate cognition: Letting nature take its course". In Robbins, P. and Aydede, M. (eds), The Cambridge Handbook of Situated Cognition. Cambridge: Cambridge University Press, 55-77.

Zhang, J. and Norman, D.A. 1994. "Representations in distributed cognitive tasks". Cognitive Science 18(1): 87-122. DOI: 10.1207/s15516709cog1801_3

\section{Author's address}

Faculty of Arts and Social Sciences

Kingston University

Penrhyn Road

Kingston upon Thames

Surrey KT1 2EE

UK

\section{About the author}

Frédéric Vallée-Tourangeau is Professor of Psychology at Kingston University. He holds a BA from McGill University, an MA from the University of Pennsylvania, and a PhD from McGill University. 